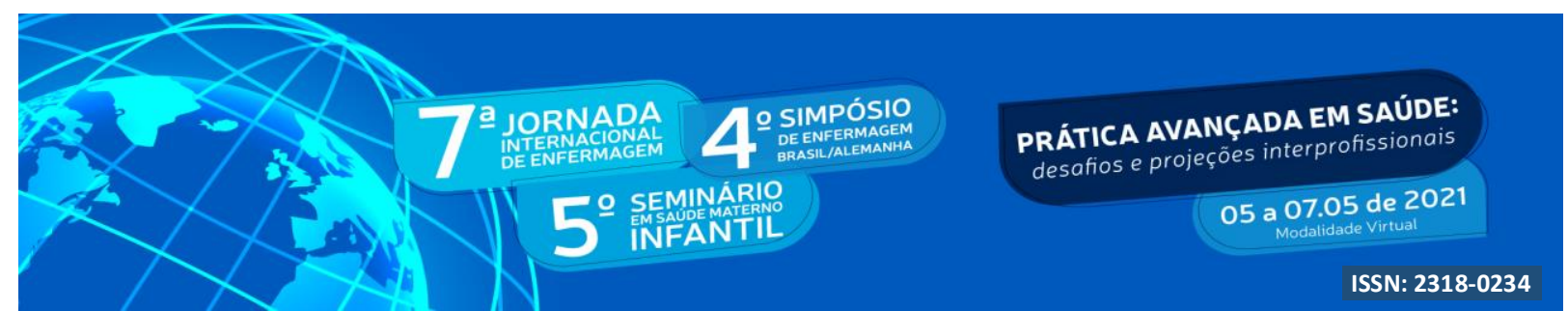

\title{
REFLEXÕES SOBRE SEGURANÇA DO PROFISSIONAL ENFERMEIRO NA ATENÇÃO PRIMÁRIA EM TEMPOS DA PANDEMIA DE COVID-19 ${ }^{1}$
}

\author{
Lívia Silveira Silva²; Selma Maria da Fonseca Viegas ${ }^{3}$
}

\begin{abstract}
RESUMO
Trata-se de um ensaio teórico com o objetivo de refletir sobre a segurança do profissional enfermeiro na Atenção Primária à Saúde em tempos de pandemia de COVID-19. Em todo território nacional as equipes da Atenção Primária responsabilizam-se por atender cerca de 80\% dos casos de COVID-19 no cotidiano dos serviços. A segurança do profissional em tempos de pandemia de COVID-19 mostra-se desfavorecida mediante às condições insalubres e inseguras de trabalho, sobrecarga física e emocional, medos, adoecimentos e óbitos de profissionais de saúde. Faz-se necessário favorecer as dimensões pessoais, culturais, sociais e os processos que envolvem a prática relacional-assistencial e a segurança do profissional, da equipe, gestores, usuários-famílias.
\end{abstract}

Palavras-chave: Atenção Primária à Saúde; Gerenciamento de Segurança; Enfermeiros.

\begin{abstract}
This is a theoretical essay with the objective of reflecting on the safety of professional nurses in Primary Health Care in times of the COVID-19 pandemic. Across the country, the Primary Care teams are responsible for dealing with about $80 \%$ of the cases of COVID-19 in the daily routine of services. Professional safety in times of pandemic of COVID-19 is shown to be disadvantaged due to unhealthy and unsafe work conditions, physical and emotional overload, fears, illnesses and deaths of health professionals. It is necessary to favor the personal, cultural, social dimensions and the processes that involve the relational-assistance practice and the safety of the professional, the team, managers, usersfamilies.
\end{abstract}

Key Words: Primary Health Care; Security Management; Nurses.

\section{INTRODUÇÃO}

A dimensão da pandemia de COVID-19 (COronaVIrus Disease-19) perpassa sobre aspectos individuais e coletivos, mutáveis e adptáveis, decisórios e limítrofes, organizacionais e culturais, finaceiros e governamentias, de vida e de morte (MAIA et al., 2019).

\footnotetext{
${ }^{1}$ Ensaio Teórico. Financiamento: Programa de Incentivo à Pós-Graduação Stricto Sensu (PIPG) da Universidade Federal de São João del rei (UFSJ).

${ }^{2}$ Enfermeira. Mestre pelo Programa de Pós-Graduação em Enfermagem da Universidade Federal de São João del-Rei, Campus Centro-Oeste (UFSJ/CCO). E-mail: livia_sjdr@ hotmail.com

${ }^{3}$ Enfermeira. Orientadora. Doutora e Pós-Doutora em Enfermagem. Docente Associado I da UFSJ/CCO, Divinópolis-MG, Brasil. E-mail: selmaviegas@ufsj.edu.br
} 


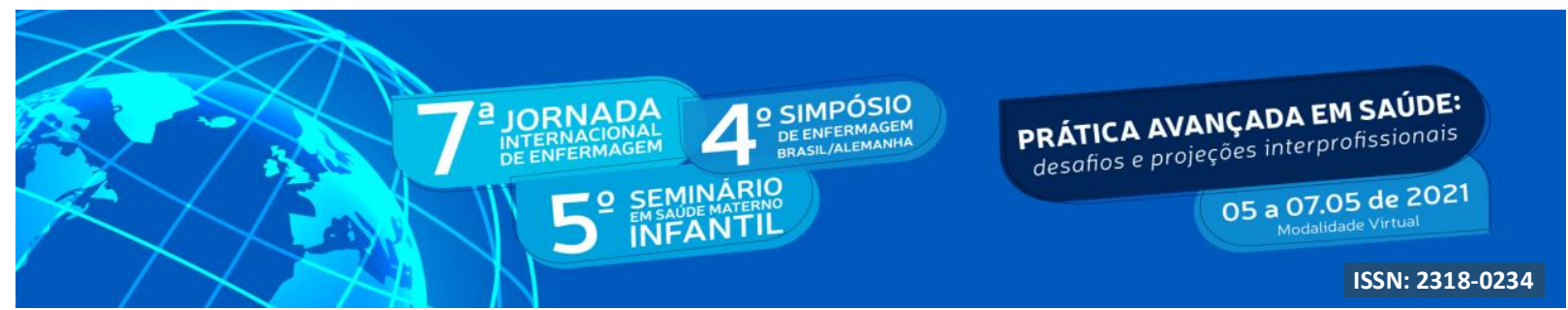

Experencia-se mundialmente novos tempos, impactando em novas condutas e cuidados em saúde, no bem-social, na economia de mercado, nos sistemas de saúde e na vida e trabalho dos profissionais de saúde na linha de frente, em uma conexão simbólica do enfrentamento árduo para salvar vidas, na efetiva atenção e magnificência do Sistema Único de Saúde (SUS) (NUNCIARONI et al., 2020) e dos demais sistemas.

Assim, faz-se necessário discutir o cotidiano, o ambiente e a ambiência, a saúde e a segurança, os desafios e as condições de trabalho, as lutas e os sentimentos dos profissionais de saúde, sobretudo, os enfermeiros da Atenção Primária à Saúde (APS) (RATES et al., 2019). A APS como uma porta de entrada no SUS, responsabiliza-se por atender cerca de 80\% dos casos de COVID-19 no cotidiano dos serviços em todo território nacional (DUNLOP et al., 2020).

Na APS, o enfermeiro tem papel significativo considerando as dimensões gerencial e assistencial do cuidado, como membro da equipe multidisciplinar da APS/Estratégia Saúde da Família (ESF), apoiando-se em conhecimentos, competências, habilidades e atitudes éticas (SANTOS et al., 2013) para possibilitar uma atuação segura, respondendo às suas necessidades como profissional, dos seus colegas de equipe, da instituição e do usuário/família (BRASIL, 2013). Além disso, para desempenhar práticas seguras, o enfermeiro precisa sentir-se seguro e protegido em seu ambiente/ambiência de trabalho, incluindo os recursos físicos, materiais e sociais que ele dispõe. Ele precisa deter conhecimentos, habilidades, técnicas e saberes para ações que assume, confiar e se corresponsabilizar pela atenção à saúde junto aos demais membros da equipe (KAHL et al., 2018).

O "ser enfermeiro na APS vivencia o protagonismo, a autonomia, a aplicabilidade de conhecimentos e as habilidades profissionais, ao desempenhar o fazer com humanização, empatia, responsabilidade e ética" (SILVA; VIEGAS; MENEZES, 2019, p. 01). O "fazer transita pela resolutividade e corresponsabilização, autonomia, humanização e acolhimento com escuta sensível, pela empatia e pelo fazer a diferença ao caminhar junto com o usuário em suas necessidades. Nesse fazer quotidiano, o enfermeiro vivencia condições de trabalho precárias" (SILVA; VIEGAS; MENEZES, 2019, p. 04). 


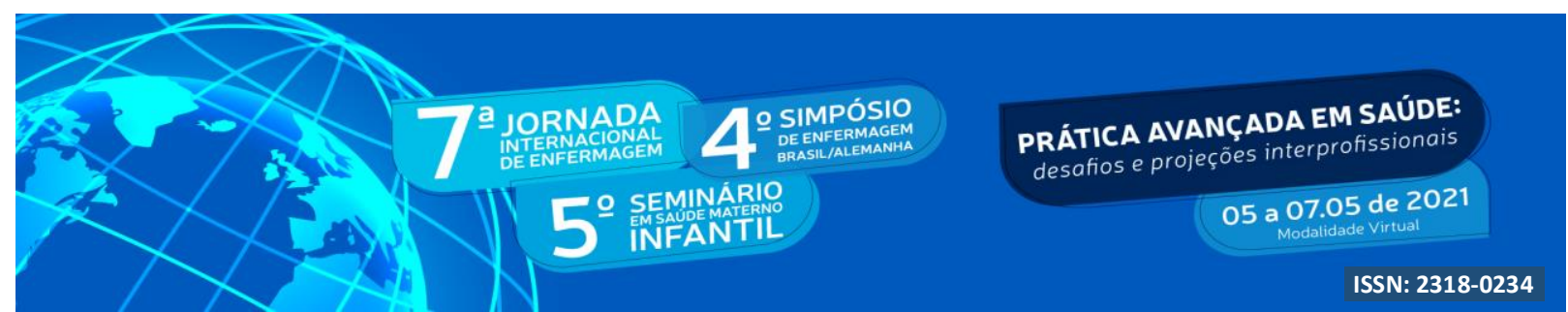

Em decorreência do cenário da pandemia e os desafios impostos aos profissionais de saúde na atenção à saúde de pessoas adoecidas pela COVID-19, a considerar os profissionais da Enfermagem, torna-se necessário voltar-se para a segurança do profissional e as dimensões e aspectos que envolvem a cultura de segurança para boas práticas.

Desarte, a dimensão do ser, considerando ideias, princípios, valores e crenças do profissional, são capazes de delinear e reflexionar o agir humano e seus propósitos para o enfrentamento desse grave problema de saúde pública. Neste cenário, a segurança do profissional se estende aos aspectos éticos e bioéticos da atenção e cuidado às pessoas, às ações profissionais individuais e em equipe, e a inter-relacão com a equipe e com os familiares (KOERICH; MACHADO; COSTA, 2005), considerando as angústias medos e anseios.

\section{OBJETIVO}

Refletir sobre a segurança do profissional enfermeiro na Atenção Primária à Saúde em tempos da pandemia de COVID-19

\section{METODOLOGIA}

O estudo caracteriza-se por um ensaio teórico acerca da segurança do profissional enfermeiro na Atenção Primária à Saúde em tempos da pandemia de COVID-19.

O ensaio constitui-se de análise e reflexões em relação ao objeto/temática abordada, independentemente de sua natureza ou característica, um método para apreender a realidade (MENEGHETTI, 2011).

Neste estudo, foi possível extrair da literatura contextos, construtos, características e significados essenciais à reflexão sobre segurança do profissional enfermeiro.

\section{PANDEMIA DE COVID-19 E A SEGURANÇA DO PROFISSIONAL ENFERMEIRO}

A segurança do profissional projeta-se a partir de elementos-chave da cultura organizacional ou de segurança, baseados, a priori, nos comportamentos e nas subjetividades - crenças, valores e atitudes - individuais ou coletivas e nos aspectos particulares, prioritários 


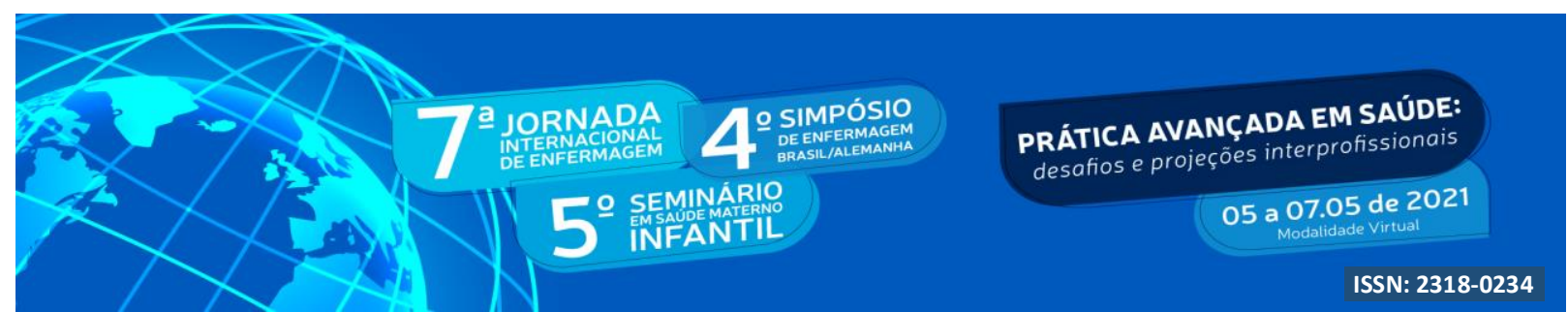

e motivadores das organizações - visão, missão, rotinas, ambiente e ambiência. Fortemente almejada, a cultura de uma organização envolve perspectivas antropológicas, psicológicas e sociais, capazes de impactar negativamente ou positivamente na qualidade e na segurança do profissional e de seu trabalho (CAMPOS; DIAS, 2012).

Na APS, a segurança do profissional pode ser favorecida ao considerar as práticas de enfermagem baseadas em evidências, o fortalecimento do diálogo, a educação permanente, a sensibilização de usuários para a corresponsabilização sanitária, e a qualificação da gestão local (MESQUITA et al., 2016; GONTIJO et al., 2020). Por conseguinte, pode ser desfavorecida perante os processos de comunicação deficientes entre usuários-profissional, profissional-profissional, profissional-gestão, dimensionamento pessoal, carência de recursos físicos, materiais e pessoais, falta de educação permanente, sobrecarga de trabalho, esgotamento profissional, (des)motivação, (des)valorização do profissional (REIS et al., 2018; GONTIJO et al., 2020).

As experiências adquiridas durante anos de trabalho, comunicação eficaz, discussão e reflexão em torno das práticas cotidianas são fatores que potencializam a segurança do profissional (SMITS et al., 2018). A experiência profissional adquirida configura-se também como base e elemento ético para ações seguras e como forte apoiadora da deliberação moral no cotidiano do profissional enfemerio e da equipe de enfermagem (RAMOS et al., 2020).

Em linhas iniciais, pode-se interpretar que o Código de Ética da Enfermagem relaciona a segurança do profissional às atividades exercidas com autonomia e segurança técnico-cientifica segundo os pressupostos éticos-bioéticos e legais da profissão e da assistência em saúde (COFEN, 2017).

Entretanto, mais do que uma proteção individual e coletiva, a segurança do profissional favorece a qualidade e a eficiência do cuidado e da assistência em saúde, por meio de processos de comunicação, diálogo, compartilhamento, reflexão e aprendizado (PICOLOTTO et al., 2019).

Contudo, o atual cenário nacional-global da pandemia de COVID-19 não mostra-se favorável à segurança do profissional: pela sobrecarga emocional e de trabalho, escassos recursos em decorrência do subfinanciamento da APS ao logo das últimas décadas, além dos entraves que esta porta de entrada enfrenta para a continuidade e longitudinalidade do cuidado 


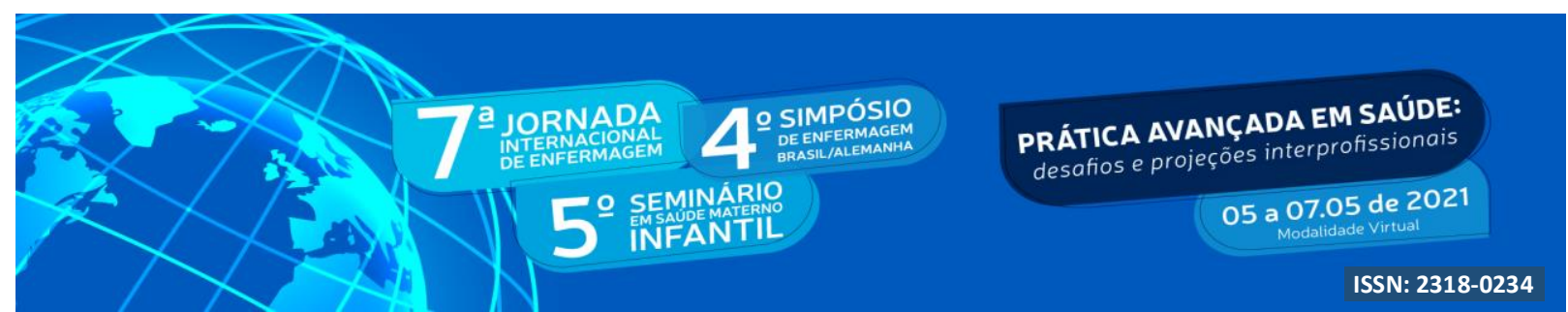

(NUNCIARONI et al., 2020); pelas fragilidades no controle da doença e insegurança frente ao contexto novo e atípico (SOUZA et al., 2021). Além das condições insalubres e inseguras de trabalho, expressas pela escassez de recursos para proteção individual, culminando em medo de se infectar ou de infectar outrém, surge o desgaste físico gerado pela sobrecarga de trabalho, os agravos psicológicos perante o adoecimento e óbitos de profissionais de saúde devido à COVID-19 (MIRANDA et al., 2020).

A realidade impõe: quem cuidará de quem cuida? Faz-se necessário favorecer as dimensões pessoais, culturais, sociais e os processos que envolvem a prática relacionalassistencial e a segurança do profissional, da equipe, gestores, usuários-famílias.

Fortalecem a segurança do profissional a efetiva comunicação, a troca de experiências/sentimentos, acolhimento e suporte emocional, a sensibilização e união dos profissionais (SOUZA et al., 2021).

Outrossim, "ao considerar a busca pela valorização e o reconhecimento social da profissão de enfermagem, torna-se perceptível a iminência da segurança do profissional, interdependente de melhores condições de infraestrutura/recursos e de trabalho, das competências e habilidades do profissional, do compromisso ético no desempenho das funções, de recursos humanos suficientes para a demanda do serviço, do apoio da gestão e de permanente educação no serviço" (VIEGAS, 2020, p.01).

O momento torna-se oportuno para reflexões necessárias e improrrogáveis sobre a segurança do profissional. Ressalta-se que diante deste cenário desafiante, o compromisso, o profissionalismo e as primazias da Enfermagem são (e sempre serão) considerados na atenção e cuidado das pessoas/famílias (QUADROS et al., 2020).

\section{CONSIDERAÇÕES FINAIS}

Este cenário pandêmico expõe as fragilidades esturturais, de recursos e de apoio necessárias à segurança do profissional, reforçando as lutas dos profissionais para visibilidade e valorização das profissões da Enfermagem. Ao esperançar mudanças e dias melhores na luta pela defesa da vida e da saúde individual e coletiva, segue a Enfermagem, profissão de grande relevância social. 


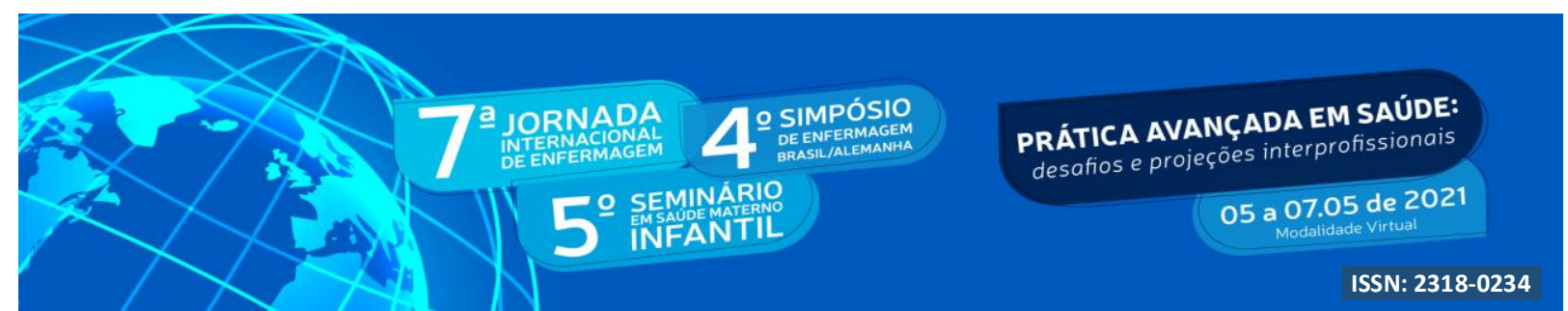

\section{REFERÊNCIAS}

BRASIL. Ministério da Saúde. Portaria $n^{\circ} 529$, de $1^{\circ}$ de abril de 2013. Institui o Programa Nacional de Segurança do Paciente (PNSP). Brasília, 2013. Disponível em:http://bvsms.saude.gov.br/bvs/saudelegis/gm/2013/prt0529_01_04_2013.html

CAMPOS, D , C.; DIAS, M. C. F. A cultura de segurança no trabalho: um estudo exploratório. Revista Eletrônica Sistemas e Gestão [online]. v.7, n.4, p. 594-604, 2012. DOI: https://doi.org/10.7177/sg.2012.V7.N4.A7

COFEN. Conselho Federal de Enfermagem. Resolução COFEN no 564/2017. Aprova o novo Código de Ética dos Profissionais de Enfermagem. Disponível em: http://www.cofen.gov.br/resolucao-cofen-no-5642017_59145.html

DUNLOP, C.; HOWE, A.; LI, D.; ALLEN, L. The coronavirus outbreak: the central role of primary care in emergency preparedness and response. BJGP Open [online]. v.4, n.1, 2020. DOI: https://doi.org/10.3399/bjgpopen20X101041

GONTIJO, M. D.; VIEGAS, S. M. F.; FREITAS, A. T. S; MAIA, A. F. F.; NITSCHKE, R. G.; NABARRO, Ml. Daily practice in the Brazilian Unified Health System in its third decade. Esc Anna Nery [online]. v.24, n.4, e20190350, 2020. DOI: 10.1590/2177-9465-EAN-20190350

KAHL, C.; MEIRELlES, B. H. S.; LANZONI, G. M. M.; KOERICH, C.; CUNHA, K. S.. Actions and interactions in clinical nursing practice in Primary Health Care.

Revista da Escola de Enfermagem da USP [online]. v.52, n. e03327, 2018. DOI: http://dx.doi.org/10.1590/S1980-220X2017025503327

KOERICH, M; SANTOS, R. R. M.; COSTA, E. The ethics and the bioethics: an initial reflection. Texto Contexto Enferm. [online]. v. 14, n. 1, 2005. DOI: http://dx.doi.org/10.1590/S0104-07072005000100014

MAIA, M. A.; PAIVA, A. C. O.; MORETÃO, D. I. C.; BATISTA, R. C. R.; ALVES, M. O cotidiano de trabalho na enfermagem: uma reflexão sobre práticas profissionais. Cienc Cuid Saude [online]. v.18, n.4, p. e43349, 2019. DOI: 10.4025/cienccuidsaude.v18i4.43349

MENEGHETTI, F. K. O que é um ensaio-teórico? Revista de administração comtemporânea [online]. v.15, n.2, p.320-32, 2011. DOI: http://dx.doi.org/10.1590/S141565552011000200010

MESQUITA, K. O.; SILVA, L. C. C.; LIRA, R. C. M.; ALINY, C.; FREITAS, S. L.; LIRA, G. V. Segurança do paciente na atenção primária à saúde: Revisão Integrativa. Cogitare Enferm [online]. v. 21, n. 2, p. 1-8, 2016. Disponível em: https://revistas.ufpr.br/cogitare/article/view/45665/28526 


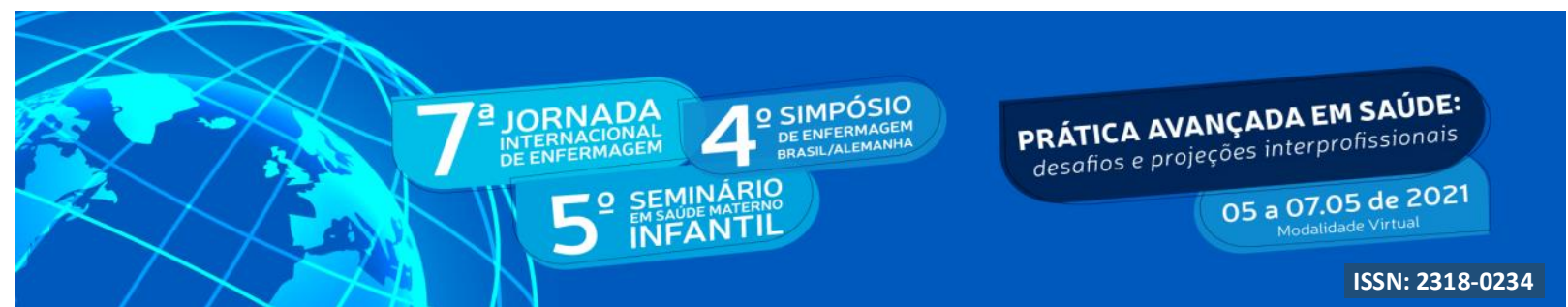

MIRANDA, F. M. A.; SANTANA, L. L.; PIZZOLATO, A. C.; SAQUIS, L. M. M. Condições de trabalho e o impacto na saúde dos profissionais de enfermagem frente a Covid19. Cogitare enferm. [online]. n. 25, p. e72702, 2020. DOI: http://dx.doi.org/10.5380/ce.v25i0.72702

NUNCIARONI, A. T.; CUNHA, F. T. S.; VARGAS, L. A.; CORREAA, V. A. F. New Coronavirus: (Re)thinking the care process in Primary Health and Nursing. Rev Bras Enferm. [online]. v. 73, n.(Suppl 2), p.e20200256; 2020. DOI: http://dx.doi.org/10.1590/0034-7167-2020-0256

PICOLOTTO, A.; BARELlA, D.; MORAES, F. R.; GASPERI, P. A Cultura de Segurança do Paciente da Equipe de Enfermagem de um Ambulatório Central. Rev Fund Care [online]. v. 11, (n. esp), p.333-338, 2019. DOI: http://dx.doi.org/10.9789/2175-5361.2019.v11i2.333338

QUADROS, A.; FERNANDES, M. T. C.; ARAUJO, B. R.; CAREGNATO, R. C. A.

Desafios da Enfermagem Brasileira no Combate da COVID-19. Enferm. foco [online]. v.11, n.(1,n.esp), p.78-83, 2020. Disponível em: http://revista.cofen.gov.br/index.php/enfermagem/article/view/3748/807

RAMOS, F. R. S.; BREHMER, L. C. F.; DALMOLIN, G. L.; SILVEIRA, L. R.; Schneider Dulcinéia Ghizoni, Vargas Mara Ambrosina de Oliveira. et al. Association between moral distress and supporting elements of moral deliberation in nurses. Rev. Latino-Am. Enfermagem [online]. v. 28, e3332, 2020. DOI: https://doi.org/10.1590/15188345.3990.3332

RATES, H.F.; CAVALCANTE, R.B.; SANTOS, R.C.; ALVES, M. Everyday life in nursing work under the Michel de Certeau's perspective. Rev Bras Enferm [online]. v.72, n. (Suppl 1), p. 341-5, 2019. DOI: http://dx.doi.org/10.1590/0034-7167-2017-0361

REIS, C.L.; TAVARES, C.S.S.; SANTANA, C.A.; MENEZES, M.O.; ANDRADE, R.X.; GOIS, R.M.O. A interface da cultura de segurança na gestão de qualidade: um estudo bibliográfico. Ciências Biológicas e de Saúde Unit [online]. v.5, n.1, p.103-116, 2018. Disponível em: https://periodicos.set.edu.br/cadernobiologicas/article/view/5315

SANTOS, J.L.G.; PESTANA, A.L.; GUERRERO, P.; MEIRELLES, B.S.H.; ERDMANN, A.L. Práticas de enfermeiros na gerência do cuidado em enfermagem e saúde: revisão integrativa. Rev. bras. Enferm [online]. v. 66, n. 2, p. 257-263, 2013. DOI: https://doi.org/10.1590/S0034-71672013000200016

SILVA, Lívia Silveira; VIEGAS, Selma Maria da Fonseca; MENEZES, Cássia. Ser enfermeiro no quotidiano da atenção primária à saúde: o fazer, o aprender e o conviver. Online Brazilian Journal of Nursing. 2019; 18(1):1-8. Disponível em: http://www.objnursing.uff.br/index.php/nursing/article/view/6351/html_2 


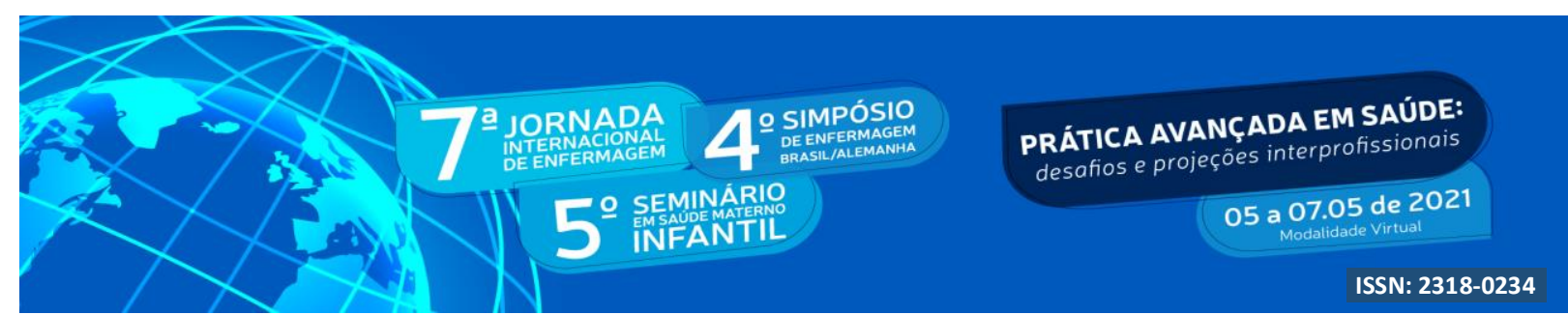

SMITS, M.; KEIZER, E.; GIESEN, P.; DEILKAS, E.C.T.; HOFOSS, D.; BONDEVIK, G.T. Patient safety culture in out-of-hours primary care services in the Netherlands: a crosssectional survey. Scand J Prim Health Care [online]. v.36, n.1, p.28-35, 2018. DOI: 10.1080/02813432.2018.1426150.

SOUZA, N.V.D.O.; CARVALHO, E.C.; SOARES, S.S.S.; VARELLA, T.C.M.M.L.; PERREIRA, S.R.M.; ANDRADE, K.B.S. Nursing work in the COVID-19 pandemic and repercussions for workers' mental health. Rev Gaúcha Enferm [online]. v.42, n.(esp), p.e20200225, 2021. DOI: http://dx.doi.org/10.1590/1983-1447.2021.20200225

VIEGAS, S.M.F. Lâmpada que não se apaga: enfermagem em prol do reconhecimento social e valorização de seus profissionais. HU Rev. v.46, p.1-2, 2020. DOI: 10.34019/19828047.2020.v46.32774 